\title{
Using a Game Development Platform to Improve Advanced Programming Skills
}

\author{
Banyapon Poolsawas ${ }^{1}$ and Winyu Niranatlamphong ${ }^{2, *}$
}

\author{
${ }^{1}$ Department of Interactive Design and Game Development, College of Creative Design and Entertainment \\ Technology, Dhurakij Pundit University, Bangkok, Thailand \\ ${ }^{2}$ Department of Digital Creative Designs, College of Creative Design and Entertainment Technology, Dhurakij \\ Pundit University, Bangkok, Thailand
}

\begin{abstract}
A computer game is one of the most efficient instructional media for improving learners' programming skills. Implementing a game development platform or a game engine as an instruction tool provides benefits to learners. This paper observes progress in the learning process, and measures how long learners take to find solutions. The participants in the experiment were divided into two groups. The first group used traditional instructional media, such as lecturing, studying class materials, and solving assigned programming assignments. On the other hand, the second group attended the lectures, similar to the first group, and used a game development platform as instructional media to solve assigned programming assignments. The results showed that the progressive skills of the Game Development Platform learners were higher, and the problem-solving time they spent was lower.
\end{abstract}

Keywords: Game Development Platform, Programming, Learning Media.

\section{INTRODUCTION}

Many innovative educators nowadays are finding the ways to incorporate the Game Development Platform into their classrooms for helping their students to develop programming language skills. The Game Development Platform in classroom has been shown to be an effective tool for improving the students' advanced programming skills (Squire and Jenkins 2003). A game engine is a software system designed to facilitate the development of computer games. As such, a game engine provides services commonly needed by the game programs, including graphics in 2D/3D, input devices and event processing; timers, object collisions.

Other advanced game engines may support the networking, physics and artificial intelligence (Pachoulakis and Pontikakis 2015). While some game development studios use their own appropriate game engine, there is still a huge market for individual developers and even larger studios requiring for a good engine to help them create their new games. (Wikipedia 2010: http://en.wikipedia.org/wiki/List_of_ game_engines). Allowing the game programmers to easily make a lot of various kinds of games, these materials are often designed to be reusable.

*Address of correspondence to this author at the Department of Digital Creative Designs, College of Creative Design and Entertainment Technology, Dhurakij Pundit University, Bangkok, Thailand; Tel: +662-954-7300 ext: 424; Fax: +662-589-9605; E-mail: winyu.nir@dpu.ac.th

JEL: C61, C73.
One of the famous game engines is "Unity" which is used for developing many types of games. The Unity is designed for handling any complicated or frequently used components in the game development. This helps simplifying a programming in the game development. Thus, the game engines present the opportunity to improve programming skills and expose the learners' potential in their advanced programming topics (Unity Technologies 2015:http://unity3d.com/).

From the concept and the above-mentioned reason supported to be a hypothesis, the Game Development Platform may build self-motivation for the basicprogramming learners from the computer games when applied in the learning media accompanying with Classroom Design. Thus, this paper specifically focuses on the improvement of the advanced programming skills. Some particular programming languages such as algorithms design and data structure are excluded.

Developed from the Unity, the Game Development Platform consisted of 25-minute theory lesson followed by 1-hour game developing practical part in each time. The last one was to find the solution of the given problem in one and a half hours. The participants in the control group studied theory lecture and the samples from traditional media; PDF or Presentation files. For the experimental group, they also did the same theory lecture but studied through the Game 4 Development Platform. Both groups were measured by the time to find the solution approaches and the scores of Pre-Test and Post-Test. 
The plan of the remainder of the paper is as follows. Section 2 considers related work. Section 3 considers objectives and hypotheses for testing. Methodology is presented in section 4 , overall operations in section 5 and discussion and conclusion in section 6 .

\section{RELATED WORK}

\subsection{Learning Media}

Media education in general is a teaching and learning tool. Everything can be used to stimulate thoughts, feelings, concerns and abilities or skills of learners so as to facilitate the process of learning. This limitation is quite broad and includes in-depth understanding of the source, the environment, human beings and the method used for the purpose of learning.

\subsection{Game Development Platform}

A set of software tools to create a game is generally referred to as a game engine or game development platform. The tools can be anything from an integrated development environment (IDE) for a programming language ( $\mathrm{C \#}$, JavaScript and $\mathrm{C}++$ ), with some basic code for starting and ending a program, to a highly developed software package with complete physics, rendering, network and artificial intelligence (Al). Game engines or Game Development Platforms help developers and programmers to develop a game application faster because developers can use implemented technical functionalities and resources in the game engine (Dan Fu, 2008). Usable features and resources in game engine are valuable for game developer. Game engine not only helps game implementer create game faster, but it also improve quality of the game and developers' programming skill.

Game Development Platform is implemented by assembling many programming libraries together. Common programming library collection for 3D game engine consists of 3D graphics, physics, collision detection and multiplayer technique or network library (Unity Technologies 2015:http://unity3d.com/). Two of the most popular game engines today, Unreal Engine 4 and Unity Game Engine have recently adopted competitive and very appealing pricing structures for individual game developers and educational use. The Unity is developed by Unity Technologies. Unity integrates a custom rendering engine with Mono, the open source implementation of Microsoft's .NET libraries. The benefits of using Unity are many when compared to Unreal Engine 4, which is used for the popular and other game engines (Pachoulakis and Pontikakis 2015). Unity uses mostly C\# or JavaScript, where the learners are 5 sufficiently apt. Which game engine offers the shortest path to a finished game depends a lot on personal preference and experience (Petridis et al. 2010).

The Unity engine comes with complete documentation with examples for its entire APIs system. This is the benefit of Unity, to increase productivity when compared to other engines such as Unreal Engine 4 or another free game development platform (Paradox, Cry Engine, Scratch and Game Salad).

The Unity is developed by Unity Technologies. Unity integrates a custom rendering engine with Mono, the open source implementation of Microsoft's .NET libraries. The benefits of using Unity are much more when compared to Unreal Engine 4, which is used for the popular and other game engines. Unity uses mostly $\mathrm{C \#}$ or JavaScript, where the learners are sufficiently apt. This game engine offers the shortest path to finish a game depending on the developers' personal preference and experiences (Pachoulakis and Pontikakis 2015). The Unity engine comes with complete documentation and examples for its entire APIs system. This is the benefit of Unity to increase productivity when compared to other engines such as Unreal Engine 4 or another free game development platform.

\subsection{Problem-based Learning}

Problem-based learning (PBL) is an approach that challenges students to learn through engagement in a real problem. Problem-based learning was firstly developed in medical field in the 1950s. It's a powerful classroom process, which uses daily-basis problems to motivate learners identifying and applying research concepts and information, working collaboratively and communicating effectively. Problem-based learning is the teaching method in which student's gains knowledge and skills by investigating and responding to the complex or dailybasis problems (Yariv 2013). Learners are engaged in rigorous, extended process by asking questions, finding resources, and applying information.

\subsection{A 2.5D Side-Scrolling Video Game}

A $2.5 \mathrm{D}$ side-scrolling game is a video game in which the gameplay action is viewed from a side-view camera angle, and the onscreen characters generally move 


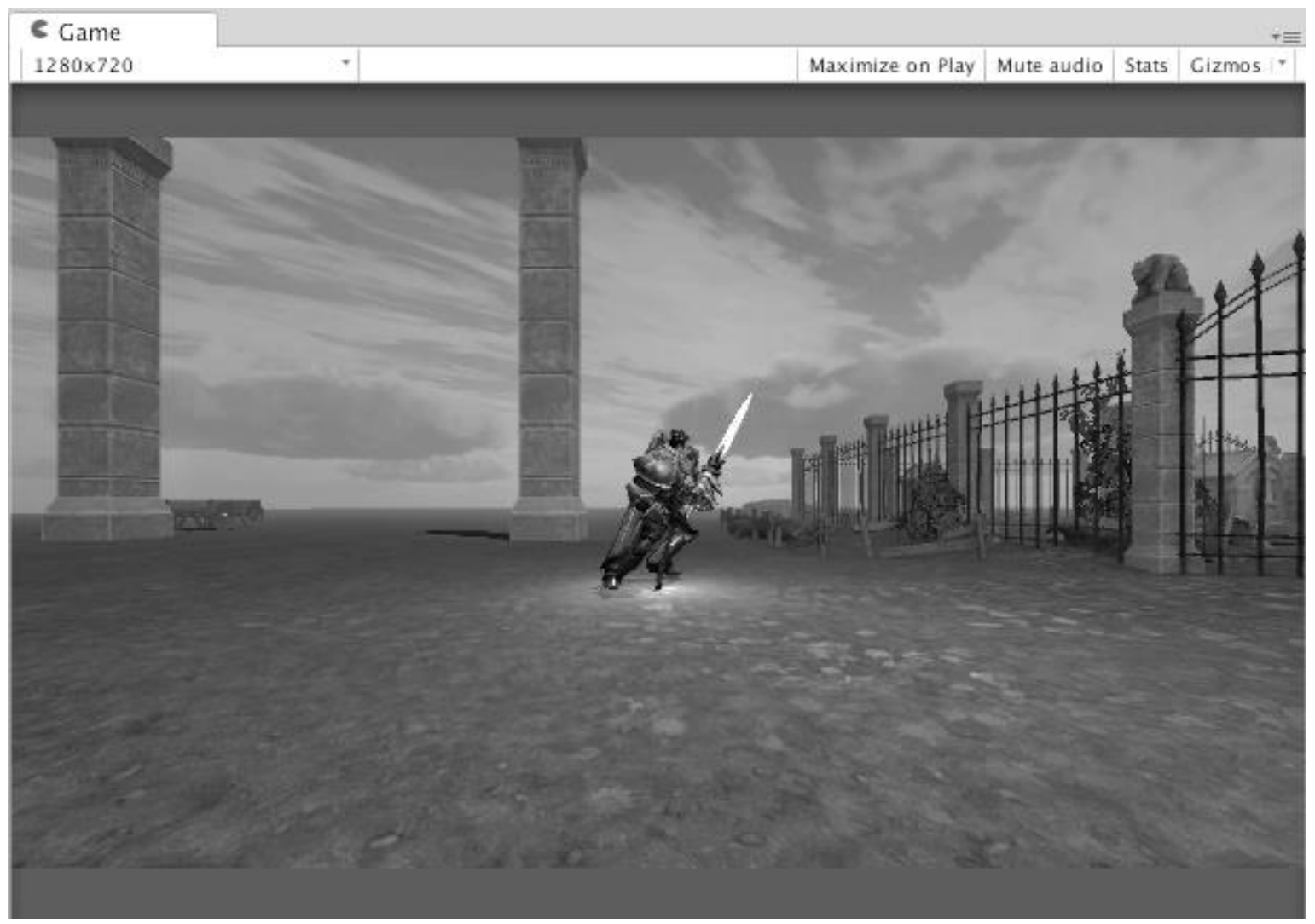

Figure 1: Trine 2 features 3D graphics, yet its gameplay is restricted to a 2D plane.

from the left side of the screen to the right to meet an objective. A term of $2.5 \mathrm{D}$ side-scrolling game that can refer to either games which feature 3D polygonal graphics but typically restrict gameplay and camera control to a 2D plane (Sharp 2014), or earlier games that attempted to simulate three-dimensional 6 graphics without the use of $3 \mathrm{D}$ polygons. $2.5 \mathrm{D}$ is used where the world layout allows for viewing only the fronts of objects. The same 3D rendering system can be used (i.e. Unity), placing the objects into a 3D world, but the world objects present a single pre-rendered texture face to the camera. Because the image is prerendered before it goes into the game, it can be made photorealistic, looking like the real object. Sample snapshots of 2.5D side-scrolling game appear in Figure 1.

\section{OBJECTIVE AND HYPOTHESIS OF THE RESEARCH}

This paper measures the progressive of the advanced programming by the test scores with the Game Development Platform which the researcher created. It shows that the average ratio is higher than the traditional methods. In addition, the learner's satisfaction with the developed tool is excellent which is made the below hypothesis:

\subsection{The First Hypothesis}

The average scores from Advanced Programming with the Game Development Platform is higher than the traditional methods included Meguigans efficiency value (Suvannatsiri and Santichaianant 2013) which the statistic hypothesis is

$\mathrm{H}_{0}=\mu_{1} \leq \mu_{2}$

$\mathrm{H}_{1}=\mu_{1}>\mu_{2}$,

$\mu_{1}$ : the average score of the Game Development Platform's learner group;

$\mu_{2}$ : the average score of the traditional methodology's learner group;

Independent variable: the Game Development Platform lesson.

Controlled variable: the learners' scores.

\subsection{The Second Hypothesis}

The learner's satisfaction through the Game Development Platform has excellent criteria which is more than 3.50 following the statistic hypothesis below: 


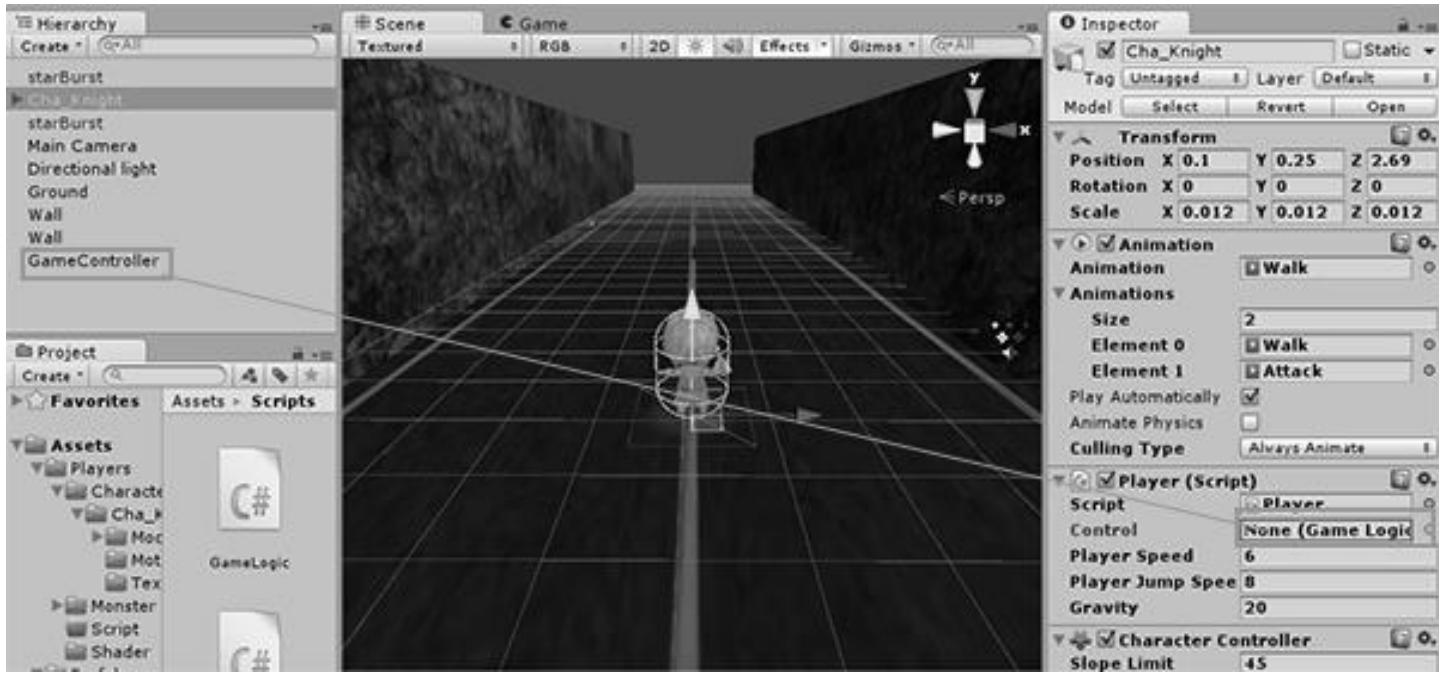

Figure 2: Student projects to solve the mathematics and physics problem with advanced programming skills via Game Development Platform.

$\mathrm{H}_{0}=\mu \leq 3.50$

$\mathrm{H}_{1}=\mu>3.50$,

$\mu$ : the average score of the learners' satisfaction through Game Development Platform;

Independent variable: the Game Development Platform lesson;

Controlled variable: the learners' satisfaction.

\section{METHODOLOGY}

This experiment would test via the analysis of the learners in the two sample groups who have similar scores. We randomly divided 15 learners each class into Experimental (E) and Control (C) groups. The first would study in the traditional instructional media as lecturing, mentioning 9 the samples through document file formats, problem solving and doing the post tests. Meanwhile, the other group attended the lecture class and use the Game Development Platform applied to find the solutions within 9 hours in 3 weeks. While the average times to solve each problem were concluded (Pe-hi and Chung 2007:446) (Yanli and Jiachen 2010:470), the average pre and post test scores were gathered at the same time (Figure 2).

\subsection{The Conceptual Framework}

As a result, the most proper framework in this research is "Pre-and-Post-Test Control Group Design".

\subsection{Population and Samples}

The 30 freshman students, the third-year students majoring from the Department of "Interactive Design and Game Development", College of Creative Design and Entertainment Technology in the first and second semester of 2015 were recruited as research participants. In addition, the students would be taught the Object-Oriented Programing Concept and Computer Programming Concept.

\subsection{Methods of Data Collection}

The "Pre-and-Post-Test Control Group Design" framework is able to effectively compare the scores of the learners better than other options which the experimental process is the below method:

$\mathrm{ER} \mathrm{O}_{1} \times \mathrm{O}_{2}$

$\mathrm{CR} \mathrm{O}_{1}-\mathrm{O}_{2}$

ER: experimental group studying through the Game Development Platform;

CR: controlled group studying through the traditional methods;

$\mathrm{O}_{1}$ : measurement or an observation with pre-test;

$\mathrm{O}_{2}$ : measurement or an observation with post-test and the period of the solution finding.

In the experiment, the 30 participants were randomly divided into two groups, which are groups with labeled " $A$ " and "B". " $A$ " is the experimental group and " $\mathrm{B}$ " is the controlled group. For the pre-test before the experiment $\left(\mathrm{O}_{1}\right)$, both participants would receive the same test format in the same surrounding. Afterwards, the class groups are separated in the 
Table 1: Results of Pre-Test and Post-Test in Experiment Group

\begin{tabular}{|c|c|c|c|c|}
\hline Achievement Test Scores & Samples & Mean (Avg.) & t-Stat & \multirow{2}{*}{3.599} \\
\hline \hline Pre-Test & 15 & 17.933 & & 2.048 \\
\hline Post-Test & 15 & 22.466 & \\
\hline
\end{tabular}

Table 2: Results of Pre-Test and Post-Test in Control Group

\begin{tabular}{|c|c|c|c|}
\hline Achievement Test Scores & Samples & Mean (Avg.) & t-Stat \\
\hline \hline Pre-Test & 15 & 17.866 & 0.77 \\
\hline Post-Test & 15 & 18.833 & 2.048 \\
\hline
\end{tabular}

different time. Before the class ended, the researcher would limit the time to find the solution for each person. When finishing all classes, the participants must do the same post-test format to measure the experiment $\left(\mathrm{O}_{2}\right)$ again.

\section{OVERALL OPERATIONS}

Comparing the learners' scores through the Game Development Platform and the traditional 12 instructional media with t-test method is found as the details in Tables 1 and 2:

The results of the Experiment Group shows the tStat of the scores was 3.599 higher than the $\mathrm{t}$-Critical average which is 2.048 meaning $\mathrm{H}_{1}$ is correct. The post-test scores are higher. The confidence level is at 0.05 showing that the learners who used the Game Development Platform are acknowledgeable about advanced programming.

From the above results, the scores of the experimental group are brought to use according to Meguigans' standard ratio through Formula 1.

Meguigans' Ratio $=\frac{M_{2}-M_{1}}{P-M_{1}}+\frac{M_{2}+M_{1}}{P}$

$M_{1}$ : average score of Pre-test;

$\mathrm{M}_{2}$ : average score of Post-test;

P: full 30 scores of the tests.
From the Table 3, it shows the Game Development Platform as a learning media is effective followed by Meguigan's standard ratio.

After comparing the scores, the satisfaction survey of the Game Development Platform was gathered in the theory of advanced programming Language, the comprehension and applying. The outcome appears that the learners achieving the goals. As shown in the Table 3, the average of the pre and post-test was 17.933 and 22.466 respectively. This apparently shows the good ratio which the tendency rises to $25.27 \%$.

\section{DISCUSSION AND CONCLUSION}

For the research of the efficiency of the learners' programming skills, the conclusion is that the samples can get the point of programing concept and develop their programming ability. Adapting the instructional media formed as the Game Development Platform and Problem-Based Learning would make the learners apply their knowledge in other sciences, students are divided to work in small groups with the guidance of a facilitator learning through solving problems and reflect on their technology experiences. The information they share together is an important part of learning as knowledge is constructed through joint efforts towards common objectives. It is proven that they can solve complex problems together. Using the Game Development Platform as a learning media in classroom is able to increase the efficiency in advanced programming skill. It also motivates, builds

Table 3: Meguigan's Score of Experiment Group

\begin{tabular}{|c|c|c|c|c|c|}
\hline Scores & Samples & Full Marks (P) & Mean (Avg.) & SD. & Meguigans' Ratio \\
\hline \hline Pre-Test & 15 & 30 & 17.933 & 2.594 & 1.722 \\
\hline Post-Test & 15 & 30 & 22.467 & 4.131 & \\
\hline
\end{tabular}






Figure 3: The satisfaction survey of the Game Development Platform was gathered in the theory of object-oriented programming.

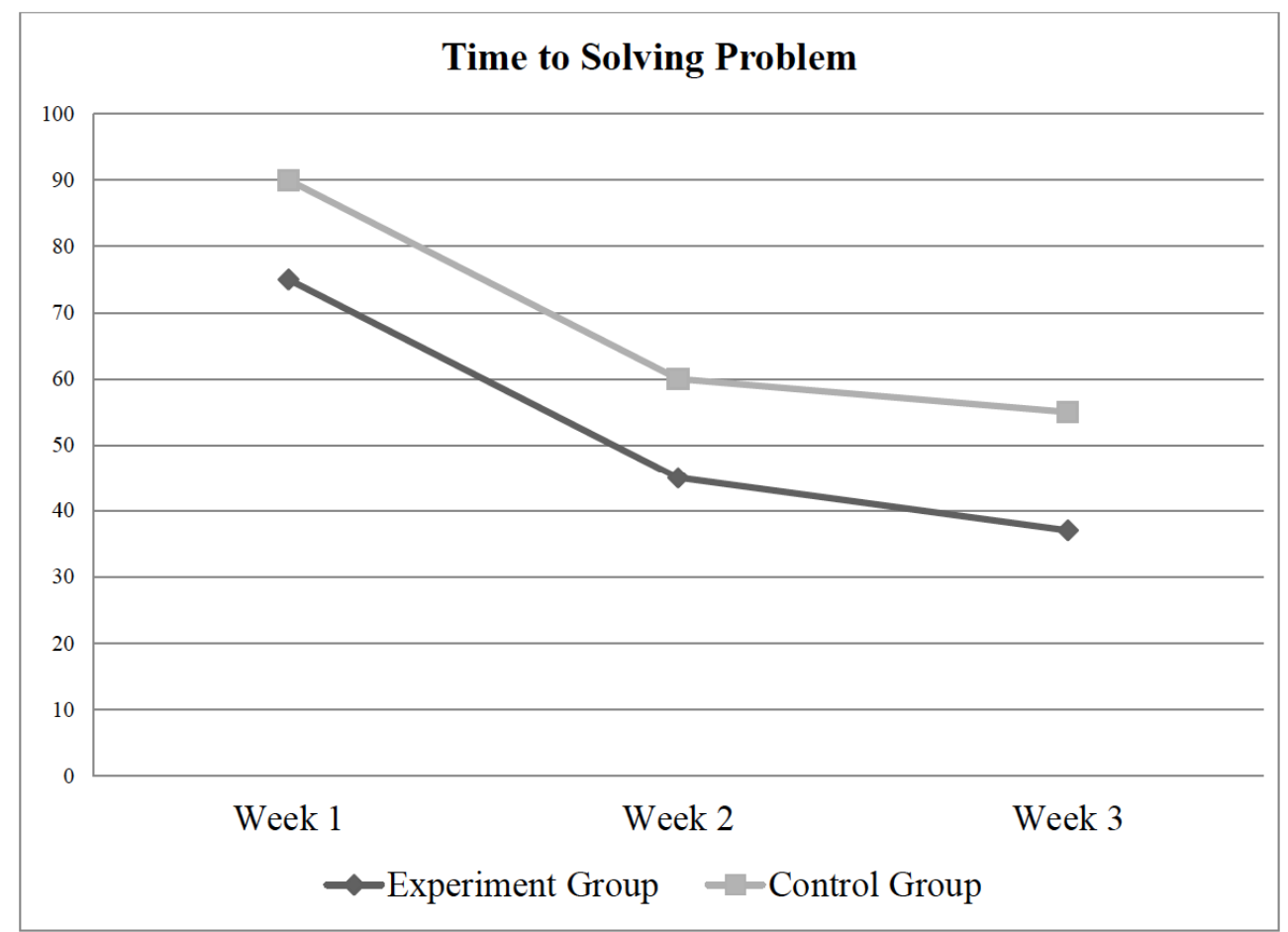

Figure 4: Shortened period to solve the problem with the Game Development Platform.

the engagement and boosts the effectiveness for the learners to solve complex problems better and faster. In the future experiments, more hypotheses can be compared with this paper to find the best educational technology.

\section{ACKNOWLEDGEMENT}

The authors wish to thank Chia-Lin Chang and Michael McAleer for helpful comments and suggestions.

\section{REFERENCES}

Game Engines 2010, Wikipedia. Retrieved November 24, 2016 (http://en.wikipedia.org/wiki/List_of_game_engines).

H.Pe-hi and M. Chung, "A Computer Adventure Game Applied in ELearning", International Conference on Intelligent Pervasive Computing, IEEE, 2007, pp. 446-451

L.Yanli and J. Zhang, "Digital Game-based College English Teaching", International Conference https://doi.org/10.1109/iccda.2010.5541038

On Computer Design And Appliations, ICCDA IEEE, 2010, pp. 470473. 
Pachoulakis, I., and G. Pontikakis. 2015. Combining features of the Unreal and Unity Game Engines to hone development skills. arXiv preprint arXiv:1511.03640.

Pachoulakis, I., and G. Pontikakis. 2015. Combining features of the Unreal and Unity Game Engines to hone development skills. arXiv preprint arXiv:1511.03640.

Pachoulakis, I., and Pontikakis, G. 2015. Combining Features of the Unreal and Unity Game Engines to Hone Development skills. arXiv preprint arXiv:1511.03640.

Petridis, P., I. Dunwell, S. De Freitas, and D. Panzoli. 2010. An engine selection methodology for high fidelity serious games. In Games and Virtual Worlds for Serious Applications (VSGAMES), 2010 Second International Conference on (pp. 2734). IEEE. https://doi.org/10.1109/vs-games.2010.26

Sharp, J. 2014. 12 DIMENSIONALITY. The Routledge Companion to Video Game Studies.
Squire, K., and H. Jenkins. 2003. Harnessing the power of games in education. Insight, 3(1), 5-33.

Suvannatsiri, R., and K. Santichaianant. 2013. A Development of Learning Innovation" Debris Flow Monitoring" with Problem Based Learning Approach: A Case Study at the Amphur Khao Phanom, Thailand. International Proceedings of Economics Development and Research, 60, 49.

Unity Technologies. Retrieved November 19, 2016 (http://unity3d.com/).

Unity Technologies. Retrieved November 27, 2016 (http://unity3d.com/).

Yariv, E. 2013. Teachers' professional experience: Solving simple and complex problems. International Journal of Educational Research, 60, 19-26. https://doi.org/10.1016/j.ijer.2013.03.009

Received on 16-02-2017

Accepted on 13-05-2017

Published on 09-06-2017

DOI: https://doi.org/10.6000/1929-7092.2017.06.33

(C) 2017 Poolsawas and Niranatlamphong; Licensee Lifescience Global.

This is an open access article licensed under the terms of the Creative Commons Attribution Non-Commercial License (http://creativecommons.org/licenses/by-nc/3.0/) which permits unrestricted, non-commercial use, distribution and reproduction in any medium, provided the work is properly cited. 